\title{
Molecular Simulation, Screening and Design of Nanoporous Metal-Organic Frameworks toward Potential Applications
}

\author{
Jianwen Jiang \\ Department of Chemical and Biomolecular Engineering \\ National University of Singapore \\ Singapore \\ chejj@nus.edu.sg
}

Metal-organic frameworks (MOFs) have emerged as a unique class of nanoporous materials and received tremendous interest over the last decade. The variation of metal oxides and the judicious choice of controllable organic linkers allow the pore size, volume and functionality of MOFs to be readily tailored in a rational manner. Consequently, MOFs provide a wealth of opportunities for molecular engineering of new nanoporous materials and have been considered as versatile candidates for many important potential applications. Nevertheless, the number of MOFs synthesized to date is extremely large, thus experimental testing alone is economically expensive and practically formidable. With rapidly growing computational resources, molecular simulation has become an indispensable tool to characterize, screen, and design MOFs. In this presentation, recent simulation studies in MOFs will be summarized toward potential applications such as methane storage, carbon capture, biofuel purification, water desalination, etc. It will be demonstrated that simulation at a molecular level can secure the quantitative interpretation of experimental observation, provide microscopic insight from bottom-up, and facilitate the development of new MOFs.

\section{References}

[1] J. W. Jiang, Ed., Metal-Organic Frameworks: Materials Modeling toward Potential Engineering Applications, Boca Raton, FL: Pan Stanford Publishing Pte. Ltd., 2015.

[2] K. Zhang, Z. J. He, K. M. Gupta, J. W. Jiang, "Computational design of 2D functional covalent-organic framework membranes for water desalination," Environmental Science: Water Research \& Technology, vol. 3, pp. 735-743, 2017.

[3] K. Zhang, Z. W. Qiao, J. W. Jiang, "Molecular design of zirconium tetrazolate metal-organic frameworks for $\mathrm{CO}_{2}$ capture," Crystal Growth \& Design, vol. 17, pp. 543-549, 2017.

[4] Z. W. Qiao, C. Peng, J. Zhou, J. W. Jiang, "High-throughput computational screening of 137953 metal-organic frameworks for membrane separation of a CO2/N2/CH4 mixture," Journal of Materials Chemistry A, vol. 4, pp. 1590415912, 2016.

[5] K. M. Gupta, K. Zhang, J. W. Jiang, "Water desalination through zeolitic-imidazolate framework membranes: significant role of functional groups," Langmuir, vol. 31, pp. 13230-13237, 2015.

[6] Y. Peng, T. Gong, K. Zhang, X. Lin, Y. Liu, J. W. Jiang, Y. Cui, "Engineering Chiral Porous Metal-Organic Frameworks for Enantioselective Adsorption and Separation," Nature Communications, vol. 5, pp. 4406, 2014.

[7] L. L. Zhang, Z. Q. Hu, J. W. Jiang, "Sorption-induced structural transition of zeolitic imidazolate framework-8: A hybrid molecular simulation study," Journal of the American Chemical Society, vol. 135, pp. 3722-3728, 2013.

[8] J. W. Jiang, "Recent Development of in silico Molecular Modeling for Gas and Liquid Separations in Metal-Organic Frameworks," Current Opinion in Chemical Engineering, vol. 1, pp. 138-144, 2012.

[9] J. W. Jiang, R. Babarao, Z. Q. Hu, "Molecular Simulations for Energy, Environmental and Pharmaceutical Applications of Nanoporous Materials: From Zeolites, Metal-Organic Frameworks to Protein Crystals," Chemical Society Reviews, vol. 40, pp. 3599-3612, 2011. 